\title{
ENSKOG-LIKE KINETIC MODELS FOR VEHICULAR TRAFFIC
}

\author{
A. Klar \\ Fachbereich Mathematik, Universität Kaiserslautern \\ Kaiserslautern, Germany \\ R. Wegener \\ Institut für Techno- und Wirtschaftsmathematik \\ Kaiserslautern, Germany
}

March 13, 1997

\begin{abstract}
In the present paper a general critisicsm of kinetic equations for vehicular traffic is given. The necessity of introducing an Enskog-type correction into these equations is shown. An Enskog-like kinetic traffic flow equation is presented and fluid dynamic equations are derived. This derivation yields new coefficients for the standard fluid dynamic equations of vehicular traffic. Numerical simulations for inhomogeneous traffic flow situations are shown together with a comparison between kinetic and fluid dynamic models.
\end{abstract}

\section{Introduction}

Traditionally there have been three types of approaches towards the modelling of traffic flow phenomena. The first and most basic one concerns microscopic or follow the leader models, modelling the actual response of single cars to their predecessor, see, e.g., $[1,2,3]$. Macroscopic models based on fluid dynamic equations have been proposed by a large number of authors, see, e.g., $[4,5,6,7,8]$. However some of these models have been subject to a considerable controversy, concerning their validity and applicability to traffic flow. Kinetic or Boltzmann-like models may present an intermediate step between the above two types of models. On the one side, they can be derived from microscopic considerations. On the other side, fluid dynamic models can be derived from kinetic traffic models as has been shown, e.g., in $[9,10,11,12]$. The first kinetic traffic models were published by Prigogine and Andrews [13], who introduced a Boltzmann-like term to account for the slowing 
down interactions (see Prigogine and Herman [9] for a review). However, they and most of their successors, see, e.g., $[14,11,10]$, treated the acceleration of the cars by means of a heuristic relaxation term. Only recently Nelson [15] succeeded in obtaining reasonable kinetic equations by using a kinetic description also for acceleration. In [16] a new model based on Nelsons approach is described and numerically investigated for space homogeneous traffic flow situations. For a survey concentrating on kinetic models, see [17].

In this paper we show that standard kinetic models are not able to deal with inhomogeneous traffic flow situations. Based on the model in [16] we introduce an Enskog type correction term into the kinetic equation. This yields a model which is able to describe correctly inhomogeneous traffic flow patterns. Moreover, we derive from the kinetic model a new fluid dynamic traffic flow equation by considerations well known from Enskogs theory of a dense gas. In particular, the coefficients in these fluid dynamic equations, like the traffic pressure, the anticipation term, and the relaxation time, are derived in this way from the kinetic model.

Up to now simulations of inhomogeneous real life traffic flow situations have only been done using microscopic and fluid dynamic models. The main advantage of fluid dynamic models is the small computation time necessary for such a simulation. Here, one should mention a special type of microscopic models, the cellular automaton models with simulation times that are similar to those of fluid dynamic models, see for example $[18,19]$. The present paper contains numerical simulations of inhomogeneous traffic situations for the kinetic and the derived fluid dynamic equations. Concerning the computation times for the kinetic equation it should be noted that kinetic models contain more variables than fluid dynamic models and less variables than microscopic ones. Therefore, one expects that computation times for the simulation of kinetic models range between those for microscopic and those for fluid dynamic models. We present a fast numerical scheme for kinetic models allowing the simulation of inhomogeneous traffic situations with reasonable computation times. Moreover, the coefficients for the fluid model are numerically evaluated for a special kinetic model. The resulting fluid equations are solved and the solutions are compared to the ones of the kinetic equation.

The paper is organized in the following way: In Section 2 a general criticism of kinetic equations concerning their applicability for inhomogeneous traffic flow situations is given. Section 3 contains an Enskog type kinetic model avoiding the difficulties mentioned in Section 2. In section 4 a derivation of fluid dynamic equations is shown yielding an Enskog correction to standard fluid dynamic models. Finally, in section 5 we describe a fast numerical scheme to solve the kinetic equation and present numerical results for kinetic and fluid equations for the case of a highway with a reduction of lanes from 3 to 2 . 


\section{Criticism of Standard Kinetic Equations for Vehicular Traffic}

The basic quantity in a kinetic modelling of vehicular traffic flow is the phase space distribution function $f(x, v, t)$. It describes the number of vehicles at $x$ and $t$ with speed $v \in[0, w]$, where $w$ denotes the maximal speed of the vehicles. The evolution of $f$ is given by a kinetic equation of the form

$$
f_{t}+v f_{x}=C(f)(x, v, t)
$$

with suitable inital and boundary values. $C$ is an operator acting on the speed distribution function $f$ and depending explicitely on the speed $v$, but on $x$ and $t$ only via $f(x, v, t)$. For a more detailed description of traffic flow on highways a multilane model describing each lane by its own distribution function $f_{\alpha}, \alpha=1, \cdots, N_{L}\left(N_{L}\right.$ : Number of lanes) is appropriate. However, for the sake of simplicity we restrict ourselves in this paper to a cumulative treatment of these distribution functions, i.e. $f=\sum_{\alpha=1}^{N_{L}} f_{\alpha}$. Classical kinetic traffic flow theories follow this strategy as well. We mention some examples:

Prigogine [9] modeled the interactions between the cars by a slowing down term and a relaxation term:

$$
C(f)=S(f)+R(f)
$$

The slowing down term

$$
S(f)(x, v, t)=(1-P) f(x, v, t) \int_{0}^{w}\left(v^{\prime}-v\right) f\left(x, v^{\prime}, t\right) d v^{\prime}
$$

describes the braking down interactions of a vehicle and depends on the probability of overtaking $P$. This term is derived in a similar fashion to the usual derivation of the Boltzmann equation in rarefied gas dynamics. Usually, $P$ was assumed to depend on the density

$$
\rho(x, t)=\int_{0}^{w} f(x, v, t) d v
$$

in a linear way, see [9],

$$
P(\rho)=1-\frac{\rho}{\rho_{m}}
$$

where $\rho_{m}$ denotes the maximal density. The introduction of a term like $P(\rho)$ represents the above mentioned cumulative treatment of the multilane effects. The relaxation term

$$
R(f)(x, v, t)=-\frac{f(x, v, t)-f_{0}(x, v, t)}{T}
$$

takes into account the acceleration of the vehicles to their desired speed. The desired speed distribution $f_{0}$ was assumed to be of the form

$$
f_{0}(x, v, t)=\rho(x, t) F_{0}(v)
$$


where $F_{0}$ is a given function not depending on $t$. The relaxation time $T$ for the acceleration interactions was also assumed to depend on $\rho$

$$
T(\rho)=\frac{\rho}{\rho_{m}-\rho}
$$

Another widely used model is due to Paveri-Fontana [11], who introduced a distribution function depending also on the desired speeds of the drivers. The following argument is not changed by doing this. We mention here also the model in [16]. It will be used as a basis of the Enskog-type model which we will develop in Section 3.

All these models have up to now mainly been used to simulate homogeneous traffic flow situations. Stationary distribution functions and fundamental diagrams, i.e. flux-density relations, have been computed. However, the influence of the streaming term has never been examined in detail. If the above equations are used for the description of inhomogeneous traffic flow situations, a serious drawback appears due to the positivity of the velocities $v$ : There is no mechanism in the equations to allow perturbations to propagate backwards in negative $x$ direction. This can be seen by the following trivial argument: Considering a full space problem the integral form of the kinetic equation is

$$
f(x, v, t)=f(x-v t, v, 0)+\int_{0}^{t} C(f)\left(x+v\left(t^{\prime}-t\right), v, t^{\prime}\right) d t^{\prime}
$$

In particular, this shows that the distribution function at $x$ and $t$ depends only on the distribution function at the values $x^{\prime} \leq x, t^{\prime} \leq t$, since $v$ is positive. A perturbation cannot propagate backwards in negative $x$-direction. In particular, traffic jams occuring for dense traffic situations are not allowed to travel backwards. This is strikingly in contrast to real traffic flow observations. The above type of kinetic equations is therefore only applicable for dilute traffic flow without backwards propagating information. We remark that the situation for kinetic equations in gas dynamics, like the Boltzmann equation, is completely different. There, the argument is obviously not true, since the velocity can assume positive and negative values.

The above remark has consequences for the connection between kinetic and fluid dynamic traffic flow equations. Formally the fluid dynamic equations can be derived from the kinetic ones by a moment procedure. However all the derived fluid dynamic equations, like for example the Lighthill Witham, Payne or other equations are able to describe backwards propagating disturbances, see [4] for the basic model. This means that one can not expect to obtain a strict derivation of fluid dynamic equations from the above kinetic equations due to their completely different behaviour in the above described situation.

In general, to describe correctly the behaviour of dense traffic with a kinetic equation and to obtain a consistent derivation of fluid dynamic equations, it is necessary to include the effects of the finite size extension of the vehicles. This can be done 
as shown in the next section in analogy to Enskogs theory for a dense gas. One obtains in this way a kinetic equation with the possibility of backwards propagating disturbances. Here one should also mention the work in [8], where the finite size requirement of the vehicles was heuristically included into a macroscopic equation.

\section{Enskog-like Vehicular Traffic Equations}

In this section we describe an Enskog type approach for kinetic vehicular traffic equations on the basis of the kinetic model developed in [16]. The procedure can obviously be adapted also for any other type of kinetic equation like the ones mentioned in the last section.

Microscopic Model:

Our kinetic model is based on the following microscopic model. Consider a car 1 at place $x_{1}$ with the velocity $v_{1}$ and its leading car 2 at $x_{2}$ with velocity $v_{2}$. Car 1 is assumed to change its velocity only in response to the leading car. If the vehicle is faster than the leading car and the headway to the leading car is becoming smaller than a certain threshold, its driver is slowing down or passing the leading vehicle. If the vehicle is slower than the leading car and the headway to the leading car is becoming larger than another threshold, the car is accelerating. Actually several different thresholds for slowing down and acceleration procedures are known. One observes, for example, thresholds based on a certain desired distance or on a minimal acceptable distance between a driver and his leading vehicle, see $[2,20]$. The thresholds in general depend on the velocity of the car and on the velocity of the leading vehicle. Let $N$ be the number of thresholds under consideration. If car 1 is crossing a threshold $i \in\{1, \ldots N\}$, i.e., if the headway $h=x_{2}-x_{1}$ is becoming larger or smaller than a certain threshold $h=H_{i}\left(v_{1}, v_{2}\right)$, then car 1 is changing its velocity to the new velocity $v . v_{1}$ and $v_{2}$ are, for each threshold $i$, out of a certain range $\Omega_{i}$ of values. The new velocity is taken on instantaneously in accordance with a certain distribution function

$$
\sigma_{i}\left(v, v_{1}, v_{2} ; \rho\right), \quad\left(v_{1}, v_{2}\right) \in \Omega_{i}
$$

Since $\sigma_{i}$ is a density function it has to fulfill

$$
\int_{0}^{w} \sigma_{i}\left(v, v_{1}, v_{2} ; \rho\right) d v=1
$$

The dependence of $\sigma_{i}$ on the local density $\rho$ allows to include the cumulative consideration of the multilane effects in the model. Moreover, $\sigma_{i}$ may also be a function of location dependent road condition parameters, e.g. the number of lanes, see the example in Section 5.

A slowing down maneuver could be given, e.g., by a function $H_{i}\left(v_{1}, v_{2}\right)=h\left(v_{2}\right)$, where $h$ represents the minimal acceptable distance to a leading vehicle with speed 
$v_{2}$. Since a car is slowing down only if its velocity is larger than that of his leading vehicle, $\Omega_{i}$ is in this case given by $\left\{\left(v_{1}, v_{2}\right) ; v_{1}>v_{2}\right\}$. In particular, one can recover the Prigogine slowing down term by setting $H_{i}=0, \sigma_{i}$ equal to

$$
\sigma_{1}\left(v, v_{1}, v_{2} ; \rho\right)=P(\rho) \delta\left(v_{1}-v\right)+(1-P(\rho)) \delta\left(v_{2}-v\right)
$$

and $\Omega_{i}=\left\{\left(v_{1}, v_{2}\right) ; v_{1}>v_{2}\right\}$.

\section{Kinetic Equation:}

One can derive now by arguments similiar to the derivation of the Boltzmann equation in gas dynamics, see [21] or [22], a kinetic equation as follows: Let $f(x, v, t)$ denote as before the phase-space density. Writing down the equation for the change of the total number of vehicles leads as usual to the kinetic equation:

$$
f_{t}+v f_{x}=\left(\frac{\delta f}{\delta t}\right)_{g}-\left(\frac{\delta f}{\delta t}\right)_{l}
$$

where $t \in[0, \infty), x \in[0, L], v \in[0, w]$, and $\left(\frac{\delta f}{\delta t}\right)_{g}$ and $\left(\frac{\delta f}{\delta t}\right)_{l}$, are gain and loss terms due to discontinuous velocity changes, respectively. We can write them as

$$
\left(\frac{\delta f}{\delta t}\right)_{g}=\sum_{i=1}^{N}\left(\frac{\delta f}{\delta t}\right)_{g}^{i},\left(\frac{\delta f}{\delta t}\right)_{l}=\sum_{i=1}^{N}\left(\frac{\delta f}{\delta t}\right)_{l}^{i}
$$

where $\left(\frac{\delta f}{\delta t}\right)_{g}^{i}$ and $\left(\frac{\delta f}{\delta t}\right)_{l}^{i}$ are describing the gain and loss terms that are due to the i-th threshold. We mention that for the numerical simulations in section 5 we will restrict to the case of two thresholds corresponding to acceleration and slowing-down interactions. The gain and loss terms are given by

$$
\begin{aligned}
& \left(\frac{\delta f}{\delta t}\right)_{g}=\sum_{i=1}^{N} \int_{\left(v_{1}, v_{2}\right) \in \Omega_{i}}\left|v_{1}-v_{2}\right| \sigma_{i}\left(v, v_{1}, v_{2} ; \rho\right) f_{2}\left(x, v_{1}, x+H_{i}\left(v_{1}, v_{2}\right), v_{2}, t\right) d v_{1} d v_{2} \\
& \left(\frac{\delta f}{\delta t}\right)_{l}=\sum_{i=1}^{N} \int_{\left(v, v_{2}\right) \in \Omega_{i}}\left|v-v_{2}\right| f_{2}\left(x, v, x+H_{i}\left(v, v_{2}\right), v_{2}, t\right) d v_{2}
\end{aligned}
$$

Here $f_{2}\left(x, v_{1}, x+d, v_{2}, t\right)$ denotes the pair distribution function of the cars and leading cars at $x$ and $x+d$, respectively and

$$
\rho=\rho(x, t)=\int_{0}^{w} f(x, v, t) d v
$$

To obtain from these equations a closed equation for $f$ we have to express $f_{2}$ by $f$. We mention here the work of Nelson [15], who introduced a correlation model for kinetic vehicular traffic equations using an assumption which he termed 'generalized vehicular chaos assumption'. A modified vehicular chaos assumption can be stated as

$$
f_{2}\left(x, v_{1}, x+d, v_{2}, t\right)=f\left(x, v_{1}, t\right) f\left(x+d, v_{2}, t\right) \tilde{k}(x, d, t)
$$


In addition we assume that $\tilde{k}$ does not depend explicitely on $x$ and $t$, but only via the local density. Then $f_{2}$ has a form similar to Enskogs theory of a dense gas:

$$
f_{2}\left(x, v_{1}, x+d, v_{2}, t\right)=f\left(x, v_{1}, t\right) f\left(x+d, v_{2}, t\right) k(d, \rho(x, t))
$$

We mention that in Enskogs theory $k$ depends on $\rho\left(x+\frac{d}{2}\right)$. In the revised Enskog theory it is a general functional of $\rho(\cdot, t)$. See $[23,22,24,25]$ for details on Enskog equations.

Defining

$$
\begin{aligned}
& C_{i}^{+}(f)(x, v, t) \\
= & \int_{\left(v_{1}, v_{2}\right) \in \Omega_{i}}\left|v_{1}-v_{2}\right| \sigma_{i}\left(v, v_{1}, v_{2} ; \rho(x, t)\right) k\left(H_{i}\left(v_{1}, v_{2}\right), \rho\right) \\
- & f\left(x, v_{1}, t\right) f\left(x+H_{i}\left(v_{1}, v_{2}\right), v_{2}, t\right) d v_{1} d v_{2} \\
& \int_{\left(v, v_{2}\right) \in \Omega_{i}}\left|v-v_{2}\right| k\left(H_{i}\left(v, v_{2}\right), \rho\right) f(x, v, t) f\left(x+H_{i}\left(v, v_{2}\right), v_{2}, t\right) d v_{2}
\end{aligned}
$$

we obtain the Enskog-like equation

$$
f_{t}+v f_{x}=\sum_{i=1}^{N} C_{i}^{+}(f)(x, v, t)
$$

This equation will be the basis for all further investigations. Reconsidering the arguments in Section 2 one obtains here

$$
f(x, v, t)=f(x-v t, v, 0)+\int_{0}^{t} \sum_{i=1}^{N} C_{i}^{+}(f)\left(x+v\left(t^{\prime}-t\right), v, t^{\prime}\right) d t^{\prime}
$$

In this case one observes, due to the definition of $C_{i}^{+}$, that the distribution function at $x, t$ depends not only on the distribution function at $x^{\prime} \leq x, t^{\prime} \leq t$ but also on the distribution function at $x^{\prime}>x$. This allows backwards propagating disturbances. In contrast to the gas dynamics case we see that the Enskog character of the equation is essential even for the description of the most basic properties of the equation like backwards propagating disturbances.

\section{Macroscopic Equations}

In this section we present a derivation of a fluid dynamic model with an Enskog-

correction term derived from the kinetic model in the last section. In particular, we 
obtain density dependent coefficients for the macroscopic equations. We reconsider the kinetic model in section 3 . For the sake of a simple notation we assume from now on that the sets $\Omega_{i}$ are disjoint with

$$
\cup_{i=1}^{N} \Omega_{i}=[0, w]^{2}
$$

Then one can define

$$
\sigma\left(v, v_{1}, v_{2} ; \rho\right)=\sigma_{i}\left(v, v_{1}, v_{2} ; \rho\right) \quad \text { and } \quad H\left(v_{1}, v_{2}\right)=H_{i}\left(v_{1}, v_{2}\right)
$$

for $\left(v_{1}, v_{2}\right) \in \Omega_{i}$. With

$$
C^{+}=\sum_{i=1}^{N} C_{i}^{+}
$$

we obtain the equation

$$
f_{t}+v f_{x}=C^{+}(f)(x, v, t)
$$

where $C^{+}$is given by

$$
\begin{aligned}
& C^{+}(f)(x, v, t) \\
= & \int_{[0, w]^{2}}\left|v_{1}-v_{2}\right| \sigma\left(v, v_{1}, v_{2} ; \rho\right) k\left(H\left(v_{1}, v_{2}\right), \rho\right) \\
& f\left(x, v_{1}, t\right) f\left(x+H\left(v_{1}, v_{2}\right), v_{2}, t\right) d v_{1} d v_{2} \\
- & \int_{0}^{w}\left|v-v_{2}\right| k\left(H\left(v, v_{2}\right), \rho\right) f(x, v, t) f\left(x+H\left(v, v_{2}\right), v_{2}, t\right) d v_{2}
\end{aligned}
$$

As a first step in the derivation of macroscopic equations we have, as usual in kinetic theory, to investigate the homogeneous kinetic equation.

Equilibrium (Homogeneous, stationary case):

The homogeneous equation for $f=f(v, t)$ is

$$
f_{t}=C(f)(v, t)
$$

with

$$
\begin{aligned}
& C(f)(v, t) \\
= & \left.\int_{[0, w]^{2}}\left|v_{1}-v_{2}\right| \sigma\left(v, v_{1}, v_{2} ; \rho\right) k\left(H\left(v_{1}, v_{2}\right), \rho\right)\right) f\left(v_{1}, t\right) f\left(v_{2}, t\right) d v_{1} d v_{2} \\
- & \int_{0}^{w}\left|v-v_{2}\right| k\left(H\left(v, v_{2}\right), \rho\right) f(v, t) f\left(v_{2}, t\right) d v_{2}
\end{aligned}
$$

For this kinetic equation $\rho=\int_{0}^{w} f(v, t) d v$ is constant in space and time. 
For the following arguments the homogeneous equation must have a one parameter family of stationary distributions $f_{e}(\rho, v)$ depending only on the density, i.e. for $\rho$ fixed we have

$$
f(v, t) \rightarrow f_{e}(\rho, v) \text { for } t \rightarrow \infty
$$

no matter what the inital distribution of the homogenous equation is. For example for the special kinetic model in section 5 this is numerically verified in [16].

Depending on the family of stationary distributions one defines the following equilibrium quantities: The mean velocity

$$
u_{e}(\rho)=\frac{1}{\rho} \int_{0}^{w} v f_{e}(\rho, v) d v
$$

the traffic pressure

$$
p_{e}(\rho)=\int_{0}^{w}\left(v-u_{e}(\rho)\right)^{2} f_{e}(\rho, v) d v
$$

the Enskog or anticipation coefficient

$$
\begin{aligned}
a_{e}(\rho)= & \int_{[0, w]^{2}}\left[v_{1}-\int_{0}^{w} v \sigma\left(v, v_{1}, v_{2} ; \rho\right) d v\right] \\
& H\left(v_{1}, v_{2}\right)\left|v_{1}-v_{2}\right| k(0, \rho) f_{e}\left(\rho, v_{1}\right) \partial_{\rho} f_{e}\left(\rho, v_{2}\right) d v_{2} d v_{1}
\end{aligned}
$$

and the interaction frequency

$$
\nu_{e}(\rho)=\frac{1}{\rho} \int_{[0, w]^{2}}\left|v_{1}-v_{2}\right| f_{e}\left(\rho, v_{1}\right) f_{e}\left(\rho, v_{2}\right) d v_{1} d v_{2}
$$

The last expression has to be corrected with a suitable term, if passing is included, see Section 5 .

Balance equations:

We start the derivation of fluid dynamic equations by multiplying the inhomogeneous kinetic equation (4) with the property $\phi(v)$ and integrating it with respect to $v$. One obtains the following set of balance equations:

$$
\partial_{t} \int_{0}^{w} \phi f d v+\partial_{x} \int_{0}^{w} v \phi f d v=\int_{0}^{w} \phi(v) C^{+}(f)(x, v, t) d v
$$

We define the density $n_{\phi}$ of the property $\phi$ as

$$
n_{\phi}=\int_{0}^{w} \phi f d v
$$


The important point in deriving fluid dynamic equations from kinetic Enskog equations is to identify clearly the flux and the source terms in the equation. The flux of $\phi$ due to the kinetic advection part is as usual

$$
q_{\phi}=\int_{0}^{w} v \phi f d v
$$

However, there is a second contribution to the flux coming from the Enskog collison term due to the finite size of the interaction thresholds. To obtain this flux we separate the Enskog interaction term into the interaction term of the homogeneous equation and the deviation from the homogeneous term:

$$
C^{+}=C-\left(C-C^{+}\right)
$$

We mention that we do not proceed here exactly as in Enskogs theory of a dense gas. The fact that the velocities are only positive requires a slightly different treatment.

Rewriting the balance equations we get then

$$
\partial_{t} n_{\phi}+\partial_{x} q_{\phi}+E_{\phi}=S_{\phi}
$$

with Enskog flux term

$$
E_{\phi}=\int_{0}^{w} \phi(v)\left[C(f)(x, v, t)-C^{+}(f)(x, v, t)\right] d v
$$

and source term

$$
S_{\phi}=\int_{0}^{w} \phi(v) C(f)(x, v, t) d v
$$

Using $\phi(v)=1$ and $\phi(v)=v$ one obtains equations for the traffic flow density

$$
n_{1}=\rho=\int_{0}^{w} f d v
$$

and the traffic flux

$$
n_{v}=\rho u=\int_{0}^{w} v f d v
$$

$u=n_{v} / n_{1}$ denotes the mean velocity. Since $S_{1}=0$ and $E_{1}=0$ we get for $\phi(v)=1$ the continuity equation

$$
\partial_{t} \rho+\partial_{x}(\rho u)=0
$$

Moreover, for $\phi(v)=v$ the acceleration equation

$$
\partial_{t}(\rho u)+\partial_{x}\left(p+\rho u^{2}\right)+E=S
$$

is obtained with

$$
p=\int_{0}^{w}(v-u)^{2} f d v
$$




$$
E=E_{v}=\int_{0}^{w} v\left[C(f)(x, v, t)-C^{+}(f)(x, v, t)\right] d v
$$

and

$$
S=S_{v}=\int_{0}^{w} v C(f)(x, v, t) d v
$$

To obtain closed equations for $\rho$ and $u$ one has to specify the dependence of $p, S$ and $E$ on $\rho$ and $u$.

Closure relations:

There are a variety of possible closure relations, which could be borrowed from gas dynamics. We restrict here to the derivation of nonviscous fluid dynamic equations. As usual, to find closure relations for the balance equations one has to use the stationary solutions of the homogeneous kinetic equation (5). All parameters of the fluid dynamic equation can be identified from these solutions. In the following $f_{e}(\rho, v)$ is the local equilibrium distribution associated to the local density $\rho=$ $\rho(x, t)$.

We set

$$
p=\int_{0}^{w}(v-u)^{2} f d v \sim \int_{0}^{w}\left(v-u_{e}(\rho)\right)^{2} f_{e}(\rho, v) d v=p_{e}(\rho)
$$

with $p_{e}$ defined in (7). The Enskog term

$$
\begin{aligned}
E= & \int_{0}^{w} v\left[C(f)(x, v, t)-C^{+}(f)(x, v, t)\right] d v \\
= & \int_{[0, w]^{2}}\left[v_{1}-\int_{0}^{w} v \sigma\left(v, v_{1}, v_{2} ; \rho\right) d v\right]\left|v_{1}-v_{2}\right| k\left(H\left(v_{1}, v_{2}\right), \rho\right) \\
& {\left[f\left(x, v_{1}, t\right) f\left(x+H\left(v_{1}, v_{2}\right), v_{2}, t\right)-f\left(x, v_{1}, t\right) f\left(x, v_{2}, t\right)\right] d v_{2} d v_{1} }
\end{aligned}
$$

is approximated in the following way:

We linearize the above expression in $H$ and substitute the stationary distributions $f_{e}$ for $f$. Using the definition of $a_{e}(\rho)$ in (8) this yields

$$
\begin{aligned}
E & \sim \int_{[0, w]^{2}}\left[v_{1}-\int_{0}^{w} v \sigma\left(v, v_{1}, v_{2} ; \rho\right) d v\right] \\
& H\left(v_{1}, v_{2}\right)\left|v_{1}-v_{2}\right| k(0, \rho) f_{e}\left(\rho, v_{1}\right) \partial_{\rho} f_{e}\left(\rho, v_{2}\right) \partial_{x} \rho d v_{2} d v_{1} \\
& =a_{e}(\rho) \partial_{x} \rho
\end{aligned}
$$

The source term is treated as for example in semiconductor theory, see, e.g., [26], by using a relaxation time approximation:

$$
S \sim-\int_{0}^{w} v \frac{\left(f-f_{e}\right)}{T_{e}(\rho)} d v=-\frac{\rho u-\rho u_{e}(\rho)}{T_{e}(\rho)}
$$

with

$$
T_{e}(\rho)=\frac{1}{\nu_{e}(\rho)}
$$


where $\nu_{e}$ and $u_{e}$ are defined in (9) and (6), respectively. Alltogether this yields fluid dynamic equations of the form

$$
\begin{gathered}
\partial_{t} \rho+\partial_{x}(\rho u)=0 \\
\partial_{t}(\rho u)+\partial_{x}\left(p_{e}(\rho)+\rho u^{2}\right)+a_{e}(\rho) \partial_{x} \rho=\frac{\rho u_{e}(\rho)-\rho u}{T_{e}(\rho)}
\end{gathered}
$$

where the quantities $p_{e}(\rho), a_{e}(\rho), u_{e}(\rho), T_{e}(\rho)$ are determined from the stationary solution of the homogeneous kinetic equations. The obtained equation looks formally similar to Paynes equation [5]. However, we have obtained in the above way coefficients that are derived from microscopic considerations, not only constants that are fitted to measured data.

\section{Remark 1:}

We remark that the term $a_{e}(\rho) \partial_{x} \rho$ is not due to the usual kinetic pressure term but only to the Enskog correction. The influence of the kinetic pressure is given by $p_{e}$.

\section{Remark 2:}

Obviously the procedure can be extended without difficulties to get for example a third equation for the variance. See [8] for such an equation. For the derivation of equations with viscous terms like in [6] a more complicated treatment is required.

\section{$5 \quad$ Numerical Results}

In this section we present a numerical solution of the kinetic equation for a special model in a space inhomogeneous situation. Moreover we evaluate explicitely the coefficients derived in the last section from the homogenous kinetic equation. The macroscopic equations with these coefficients are solved and the results are compared to the kinetic solution.

\section{Kinetic model:}

Before describing the discretization scheme for the kinetic equation we state briefly the explicit example for the kinetic model that we use for the simulation (see [16] for more details). Consider equation (2) with two thresholds $(N=2)$, one for slowing down and one for acceleration interactions. The slowing down threshold is given by a headway

$$
H_{1}\left(v_{1}, v_{2}\right)=h
$$

where $h$ is a positive constant. For slowing down $\Omega_{1}$ is given by

$$
\Omega_{1}=\left\{\left(v_{1}, v_{2}\right) \in[0, w]^{2} ; v_{1}>v_{2}\right\}
$$

The slowing down term is modeled by a Prigogine-like term. However we do not restrict to slowing down to the actual speed of the leading vehicle as in the Prigogine 
work, but to a range of speeds smaller than this one. We allow slowing down of car 1 to a velocity $v \in\left[\beta v_{2}, v_{2}\right] . \beta$ is some positive constant smaller than 1 . In this range a uniform distribution of velocities is assumed due to the lack of a more precise knowledge.

If the following car is faster than the leading one, we have - alternatively to slowing down - also to take into account the possibility of passing with a certain probability $P$ analogous to the Prigogine model in Section 2. We assume the velocity of the passing car to remain the same as before. This gives

$$
\sigma_{1}\left(v, v_{1}, v_{2}, \rho\right)=P \delta\left(v_{1}-v\right)+(1-P) \frac{1}{v_{2}(1-\beta)} \chi_{\left[\beta v_{2}, v_{2}\right]}(v)
$$

where $\delta(v)$ is the delta function at $v$ and $\chi_{[a, b]}$ is the characteristic function of the interval $[a, b]$.

The probability of passing $P$ depends on $\rho(x, t)$ like

$$
P=1-\frac{\rho}{\rho_{m}}
$$

where $\rho_{m}$ denotes the maximal density, which is given by the number of lanes. This is the same dependence as in the Prigogine model. $\rho_{m}$ is a location dependent road condition parameter.

The acceleration threshold is assumed to be given by the same constant

$$
H_{2}\left(v_{1}, v_{2}\right)=h
$$

For a more consistent microscopic model one would have to choose this constant larger than the constant of the slowing down threshold. However, for the kinetic model the limit case of equal constants does not yield any problems. For acceleration $\Omega_{2}$ is given by

$$
\Omega_{2}=\left\{\left(v_{1}, v_{2}\right) \in[0, w]^{2} ; v_{1}<v_{2}\right\}
$$

We assume that car 1 accelerates from its actual speed $v_{1}$ to a certain range of speeds between $v_{1}$ and $v_{1}+\alpha\left(w-v_{1}\right)$. Moreover, we assume again due to the lack of a more precise knowledge that the velocity after acceleration is uniformly distributed in the range $\left[v_{1}, v_{1}+\alpha\left(w-v_{1}\right)\right]$. This leads to the distribution function

$$
\sigma_{2}\left(v, v_{1}, v_{2} ; \rho\right)=\frac{1}{\alpha\left(w-v_{1}\right)} \chi_{\left[v_{1}, v_{1}+\alpha\left(w-v_{1}\right)\right]}(v)
$$

Since for dense traffic the possibility of acceleration is more restricted than for traffic flow with a low density, $\alpha$ is supposed to depend on the density $\rho(x, t)$ in the following way:

$$
\alpha=\alpha_{0}\left(1-\frac{\rho}{\rho_{m}}\right)
$$


where $\alpha_{0} \leq 1$ is some positive constant. Note that this model fulfills condition (3). Using the notation at the beginning of Section 4 one can define $\sigma$ by $\sigma_{1}$ and $\sigma_{2}$. Since a completely satisfying correlation model is not available, we assume for the simulation that $k(h, \rho)$ is equal to 1 .

This model is now numerically investigated.

We treat the kinetic equation by a discretization scheme, that is described in the following: A simple standard discretization of the equation in velocity-space needs a large number of discretization points in order to describe correctly the influence of the singularities appearing at $v=0$ and $v=w$. Therefore we divide the velocity space into a certain number of cells and calculate the transition rates between the cells given by the above kinetic equation. Setting $w=1$ we introduce gridpoints

$$
x_{i}=i \Delta x, \quad i=0, \ldots, M, \quad \Delta x=\frac{L}{M}, \quad v_{j}=\frac{j+\frac{1}{2}}{N}, \quad j=0, \ldots, N-1
$$

and the values $f_{i, j}=f\left(x_{i}, v_{j}, t\right)$. The discretized Enskog equation is then given by

$$
\partial_{t} f_{i, j}+v_{j} \frac{\left(f_{i, j}-f_{i-1, j}\right)}{\Delta x}=\frac{1}{N^{2}} \sum_{k, l=0}^{N-1} \bar{\sigma}_{i, j, k, l}\left|v_{k}-v_{l}\right| f_{i, k} \tilde{f}_{i, l}^{h}-\frac{1}{N} f_{i, j} \sum_{k=0}^{N-1}\left|v_{j}-v_{k}\right| \tilde{f}_{i, k}^{h}
$$

with

$$
\tilde{f}_{i, j}^{h}=(1-\alpha) f_{i+\bar{i}, j}+\alpha f_{i+\bar{i}+1, j}
$$

where

$$
\bar{i}=\max \left(i: i<\frac{h}{\Delta x}\right), \alpha=\frac{h}{\Delta x}-\bar{i}
$$

and

$$
\bar{\sigma}_{i, j, k, l}=N^{3} \int_{(j, k, l) / N}^{(j+1, k+1, l+1) / N} \sigma\left(v, v_{1}, v_{2} ; \rho_{i}\right) d v d v_{1} d v_{2}
$$

where $\bar{\sigma}_{i, j, k, l}$ is evaluated analytically. The density used to compute $\sigma$ is calculated by

$$
\rho_{i}=\frac{1}{N} \sum_{j=0}^{N-1} f_{i, j}
$$

The time derivative is treated in a standard way. This means, we implement a discrete velocity approximation of the kinetic equation using the discrete transition rates $\bar{\sigma}_{i, j, k, l}$ which are averaged values for each cell. The most important fact about this discretization is that the discrete version of the continuity equation is automatically fulfilled, since

$$
\frac{1}{N} \sum_{j=0}^{N-1} \bar{\sigma}_{i, j, k, l}=1
$$

The scheme needs only a very low number - approximately 10 cells - in velocity space to yield resonable results. 
Fluid dynamic model:

The fluid dynamic equation is solved with a splitting algorithm treating the flux and the relaxation part in separate steps. The flux part is, as usual, written in conservative form and then treated by a conservative scheme. We have simply choosen the Lax-Friedrichs algorithm.

To obtain the coefficients for the fluid dynamic equations we have to compute the stationary distributions of the homogeneous kinetic equation (5). In the present case the interaction frequency leading to a relaxation to the stationary distribution must be corrected, as already mentioned in Section 4, due to the inclusion of the possibility of passing. We get

$$
\begin{array}{r}
\nu_{e}(\rho)=\frac{1}{\rho} \int_{[0, w]^{2}}\left|v_{1}-v_{2}\right| f_{e}\left(\rho, v_{1}\right) f_{e}\left(\rho, v_{2}\right) d v_{1} d v_{2} \\
-\frac{P(\rho)}{\rho} \int_{\Omega_{2}}\left|v_{1}-v_{2}\right| f_{e}\left(\rho, v_{1}\right) f_{e}\left(\rho, v_{2}\right) d v_{1} d v_{2}
\end{array}
$$

To solve the homogenous equation we use a scheme similar to the one described in [16]. To obtain a high accuracy for the coefficients an adaptive discretization of the velocity space is introduced additionally. The parameters in the kinetic model are choosen as $\alpha_{0}=0.3$ and $\beta=0.3$. Figures 1 to 4 show plots of the quantities $u_{e}(\rho), p_{e}(\rho), \nu_{e}(\rho), a_{e}(\rho)$. The plot of the mean velocity fits quantitatively to measured data reported in [27]. At this point one should mention that the obtained coefficients depend on the microscopic model via the kinetic equation. They can not be supposed to be the correct ones for any traffic flow situation.

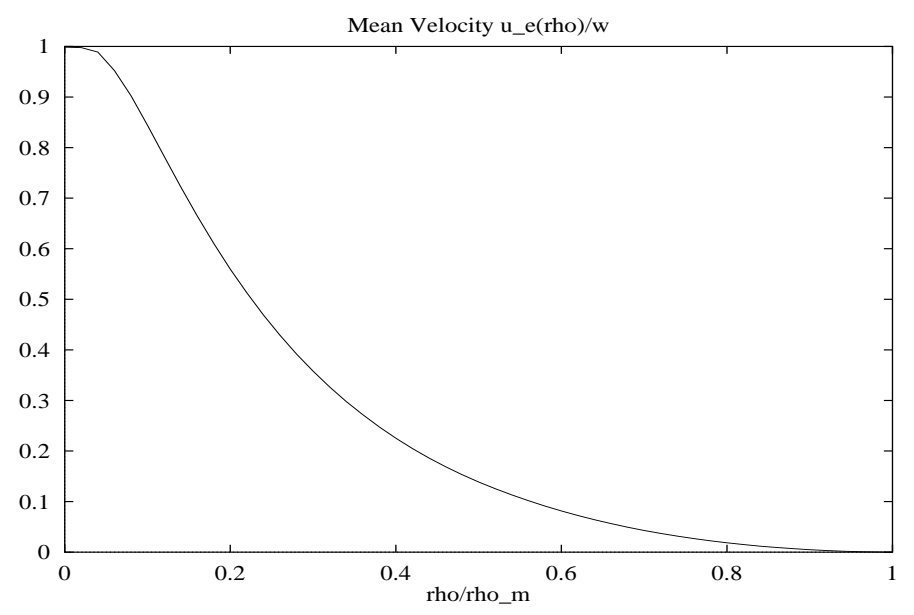

Figure 1: Mean velocity 


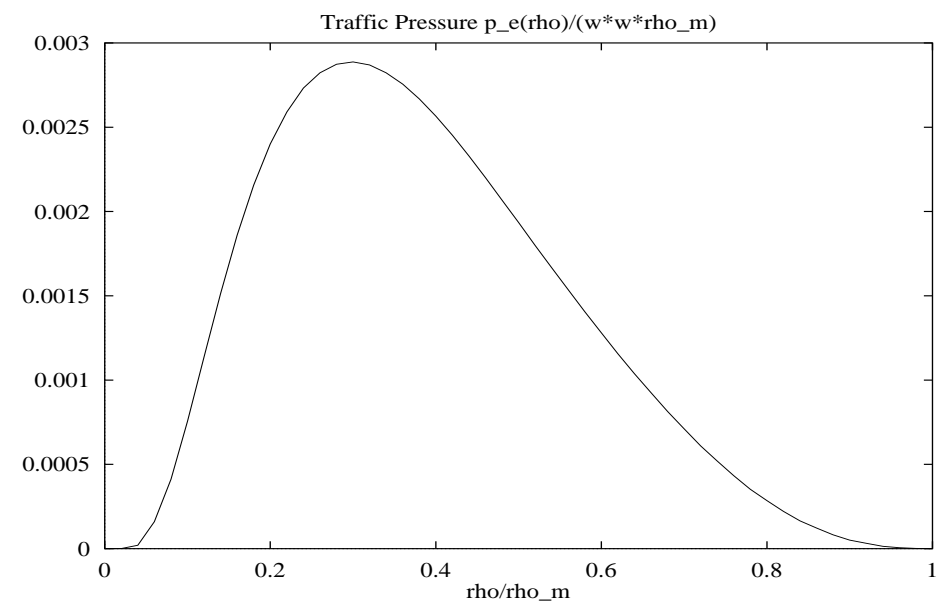

Figure 2: Traffic Pressure

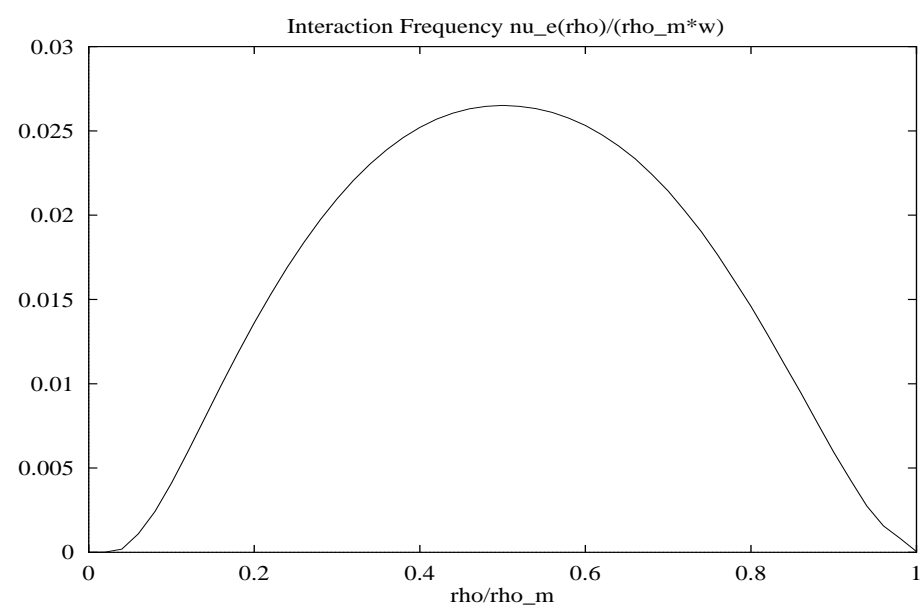

Figure 3: Interaction frequency 


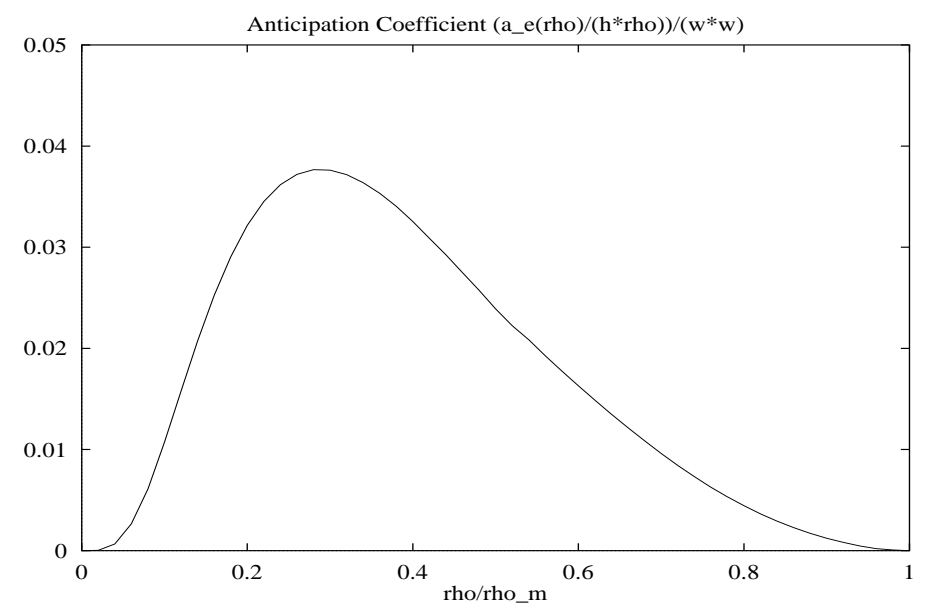

Figure 4: Anticipation coefficient

An inhomogeneous traffic flow situation:

In the following series of figures an inhomogeneous traffic flow situation is shown. We consider a highway with a reduction of the number of lanes from 3 to 2 after two thirds of the highway under consideration. The example is calculated with the kinetic equation and the derived fluid dynamic equations with the coefficients computed above. The reduction of the lanes is simply achieved by changing the maximal density $\rho_{m}$.

The units in the following calculations are given by setting the maximal velocity $w$ equal to 1 and the bumper to bumper distance $h_{0}$ equal to 1 . This yields the time unit to be equal to the bumper to bumper distance divided by the maximal velocity and the maximal density $\rho_{m}$ equal to the number of lanes under consideration. The length $L$ of the highway under consideration is equal to 1000. The space and time discretizations are $\Delta x=1, \Delta t=0.5$ for the kinetic equation and $\Delta x=0.5, \Delta t=$ 0.125 for the fluid dynamic equation. For the kinetic equation the number of points for the velocity discretization is choosen as $N=40$ and the threshold value as $h=5$.

We start with an "empty" highway and prescribe the incoming distribution function for the kinetic equation. A constant number of incoming vehicles is used. The solution of the kinetic equation, i.e the space-speed distribution of the vehicles, is shown for different times in Figures 5 to 7 . Starting with an empty highway one observes in Figure 5 free flow of the vehicles until the stretch is completely filled with vehicles. The overall density is small compared to the maximal density, such that there is no influence of the bottleneck. When the stretch finally is filled with vehicles, the speed distribution drops at the bottleneck reflecting the formation of a traffic jam as seen in Figure 6. Two modes of the distribution function are appearing at the beginning of the bottleneck. In Figure 7 the jam is running backwards. In the bottleneck itself the speed distribution recreates showing normal traffic flow with lower density. 


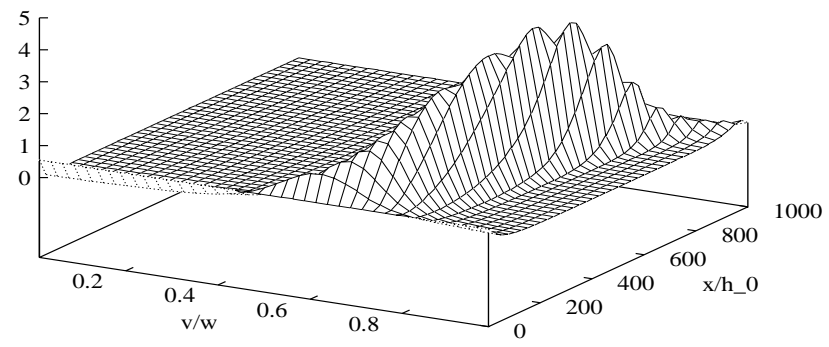

Figure 5: Space speed distribution for $t=1000$

$\mathrm{F}(\mathrm{x}, \mathrm{v})$

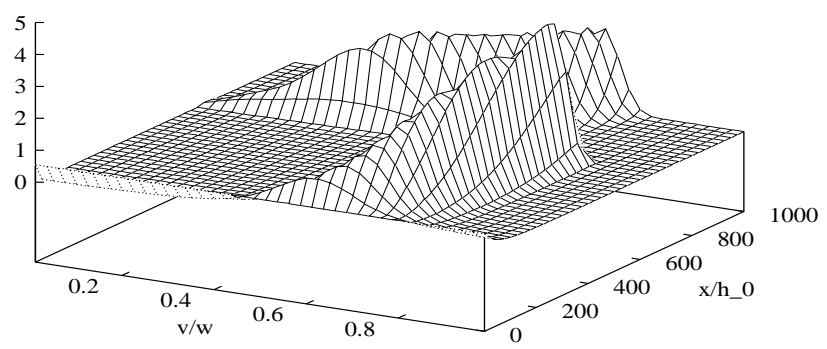

Figure 6: Space speed distribution for $t=2000$ 


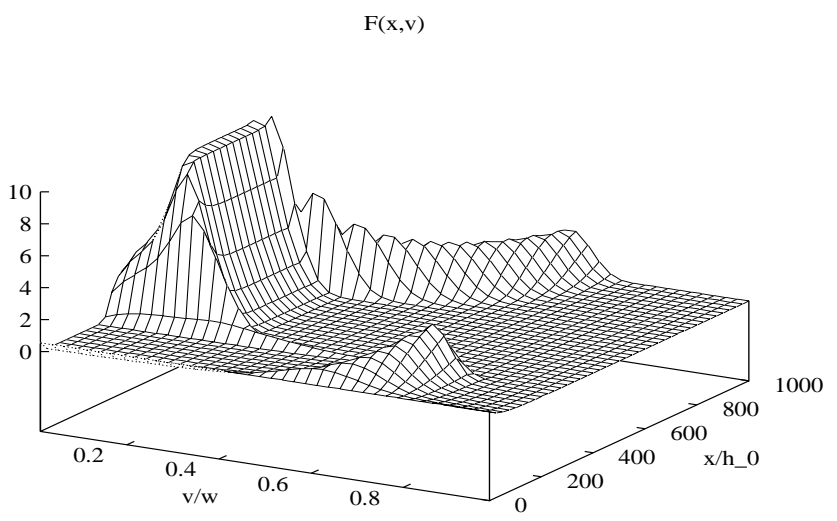

Figure 7: Space speed distribution for $t=8000$

Figures 8 and 9 show the time development of the density of vehicles for the fluid dynamic and kinetic equations.

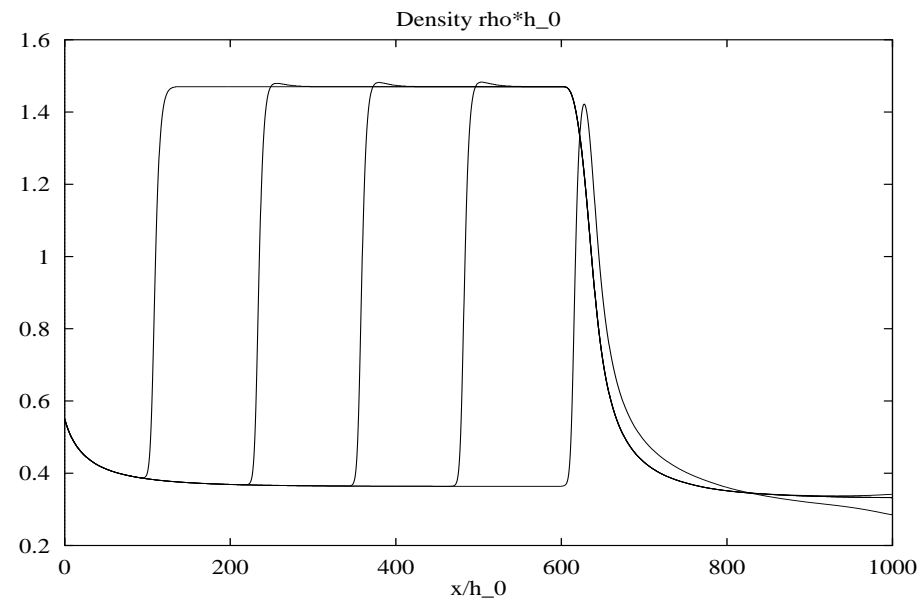

Figure 8: Time development of the density for the fluid dynamic equation at $t=$ $2000,4000,6000,8000,10000$ 


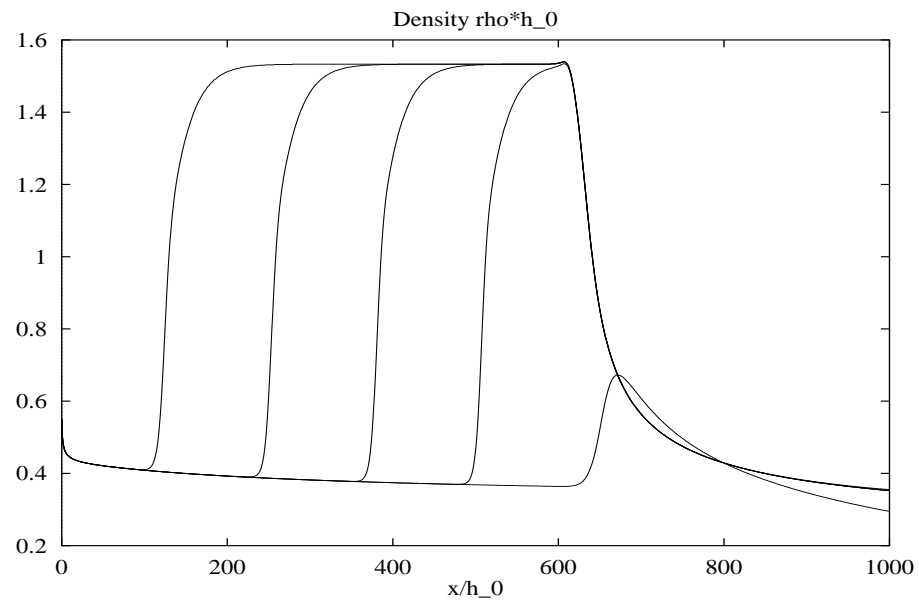

Figure 9: Time development of the density for the kinetic equation at $t=$ $2000,4000,6000,8000,10000$

We mention that, as expected, both equations show very similar behaviour. However, in the kinetic simulation the shocks are smeared out much more than in the fluid dynamic one. This is similar to the situation in the kinetic theory of gases.

Finally in Figure 10 the influence of the Enskog factor is shown. The density of the kinetic solution with $h=0$ (no Enskog correction) is plotted at time $t=2300$. The case $h=5$ is shown for comparison. In particular, one observes the explosion of the solution of the kinetic equations without the Enskog correction shortly after the time $t=2300$ and no backwards running traffic jam is observed. This is in accordance with the considerations in Section 2.

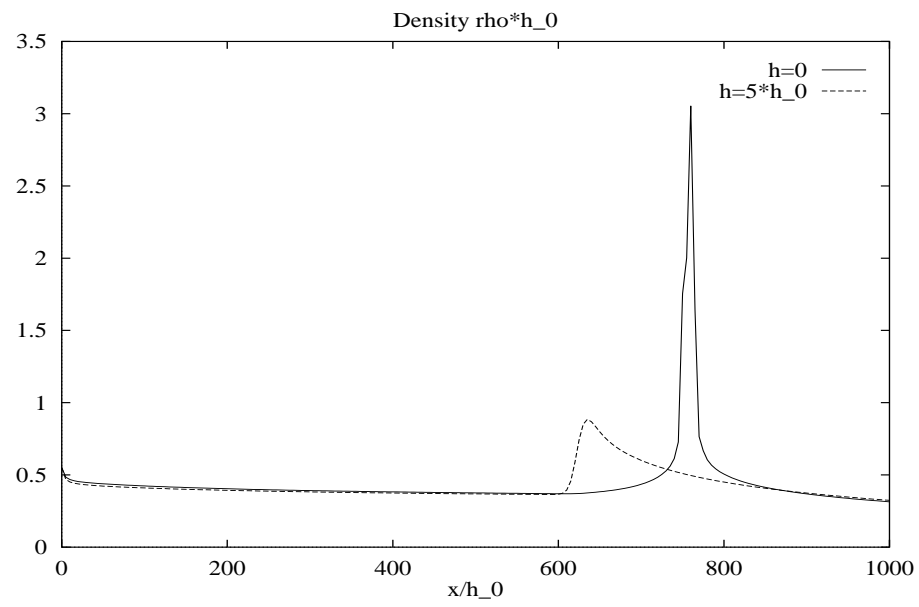

Figure 10: Density for the kinetic equation without Enskog correction 


\section{Acknowledgements}

This work was supported by a grant under the program 'Wirtschaftsnahe Forschung' (Ministry of Economy, Rheinland Pfalz, Germany). We are grateful to C. Cercignani, H. Kühne and H. Neunzert for interesting discussions and informations.

\section{References}

[1] D.C. Gazis, R.Herman, and R. Rothery. Nonlinear follow-the-leader models for traffic flow. Opns. Res., 9:545, 1961.

[2] R. Wiedemann. Simulation des Straßenverkehrsflusses. Schriftenreihe des Instituts für Verkehrswesen der Universität Karlsruhe, 1974.

[3] D.L.Gerlough and M.J. Huber. Traffic Flow Theory. Transportation Research Board Special Report 1965, Washington D.C., 1975.

[4] G.B. Witham. Linear and Nonlinear Waves. Wiley, New York, 1974.

[5] H.J. Payne. Transportation Research Record, 722:68, 1979.

[6] R.D. Kühne. Macroscopic freeway model for dense traffic. In N. Vollmuller, editor, 9th Int. Symp. on Transportation and Traffic Theory, page 21, 1984.

[7] B.S. Kerner and P.Konhäuser. Physical Review E, 50:54, 1994.

[8] D. Helbing. Improved fluid dynamic model for vehicular traffic. Physical Review E, 51:3164, 1995 .

[9] I. Prigogine and R. Herman. Kinetic Theory of Vehicular Traffic. American Elsevier Publishing Co., New York, 1st edition, 1971.

[10] W.F. Phillips. Kinetic Model for Traffic Flow. National Technical Information Service, Springfield, Virginia, 1977.

[11] S.L. Paveri-Fontana. On Boltzmann like treatments for traffic flow. Transportation Research, 9:225, 1979.

[12] D. Helbing. Gas-kinetic derivation of Navier-Stokes-like traffic equation. Preprint, University of Stuttgart, 1995.

[13] I. Prigogine and F.C. Andrews. A Boltzmann like approach for traffic flow. Oper. Res., 8:789, 1960.

[14] M. Lampis. Transportation Science, 12:16, 1978.

[15] P. Nelson. A kinetic model of vehicular traffic and its associated bimodal equilibrium solutions. Transp. Theory and Stat. Phys., 24:383, 1995. 
[16] R. Wegener and A. Klar. A kinetic model for vehicular traffic derived from a stochastic microscopic model. to appear in Transp. Theory Stat. Phys., 1996.

[17] A. Klar, R.D. Kühne, and R. Wegener. Mathematical models for vehicular traffic. to appear in Surv. Math. Ind., 1996.

[18] K. Nagel and A. Schleicher. Parallel Computing, 20:125, 1994.

[19] M. Schreckenberg, A. Schadschneider, K. Nagel, and N. Ito. Physical Review E, 51:2939, 1995 .

[20] N. Anstett. Entwicklung eines ereignisorientierten Fahrzeug-FolgeModells zur mikroskopischen Verkehrssimulation. Diplomarbeit Universität Stuttgart/Daimler Benz AG, 1992.

[21] C. Cercignani. The Boltzmann Equation and its Applications. Springer, New York, 1st edition, 1988.

[22] J.H. Ferziger and H.G. Kaper. Mathematical theory of transport processes in gases. North Holland, Amsterdam, 1972.

[23] C. Cercignani and M. Lampis. On the kinetic theory of a dense gas of rough spheres. J. Stat. Phys., 53:655-672, 1988.

[24] J. Lebowitz, J. Percus, and J. Sykes. Phys. Rev., 188:487, 1967.

[25] P. Resibois. H-theorem for the (modified) nonlinear enskog equation. J. of Stat. Phys., 19:593, 1978.

[26] P. Markowich, C. Ringhofer, and C. Schmeiser. Semiconductor equations. Springer, New York, 1990.

[27] R.D. Kühne and Rödiger. Proceedings of the 1991 Winter Simulation Conference, Phoenix, Arizona, 1991. 


\section{List of Figures}

1 Mean velocity .......................... 15

$2 \quad$ Traffic Pressure . . . . . . . . . . . . . . . 16

3 Interaction frequency . . . . . . . . . . . . . 16

$4 \quad$ Anticipation coefficient . . . . . . . . . . . . 17

$5 \quad$ Space speed distribution for $t=1000 \ldots \ldots \ldots \ldots$

$6 \quad$ Space speed distribution for $t=2000 \ldots \ldots \ldots \ldots$

$7 \quad$ Space speed distribution for $t=8000 \ldots \ldots \ldots \ldots \ldots$

8 Time development of the density for the fluid dynamic equation at $t=2000,4000,6000,8000,10000 \ldots \ldots \ldots \ldots \ldots$

9 Time development of the density for the kinetic equation at $t=$ $2000,4000,6000,8000,10000 \ldots \ldots \ldots 20 \ldots \ldots$

10 Density for the kinetic equation without Enskog correction . . . . 20 
Keywords:

Traffic Flow, Enskog Equation, Boltzmann Equation, Fluid Dynamic Models, Payne Equation, Simulation of Inhomogeneous Traffic Flow 
Mean Velocity u_e(rho)/w

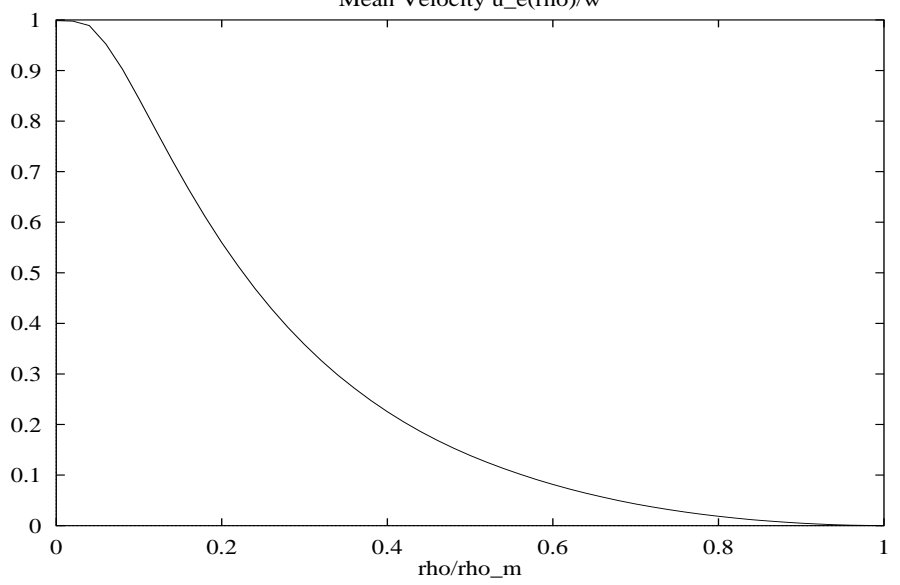




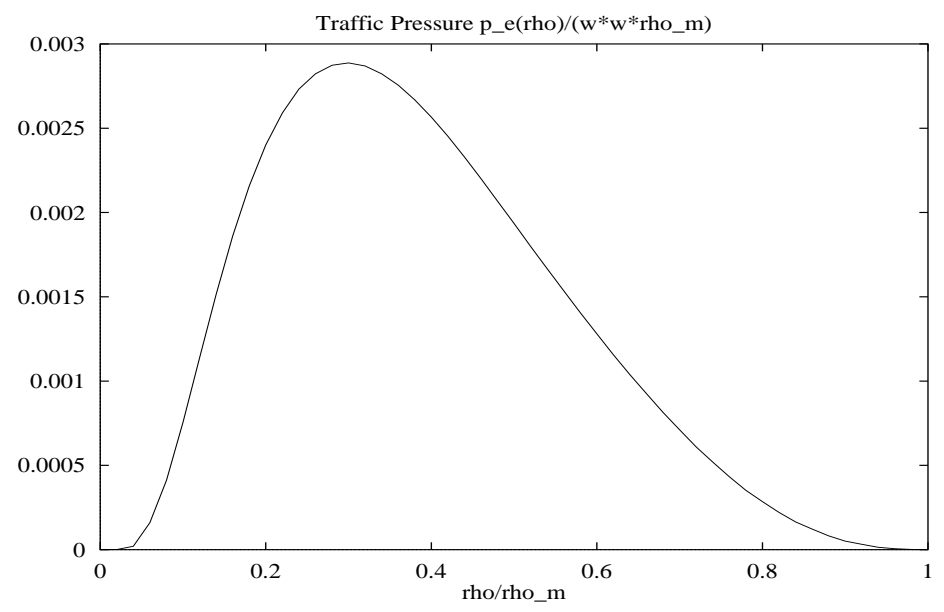




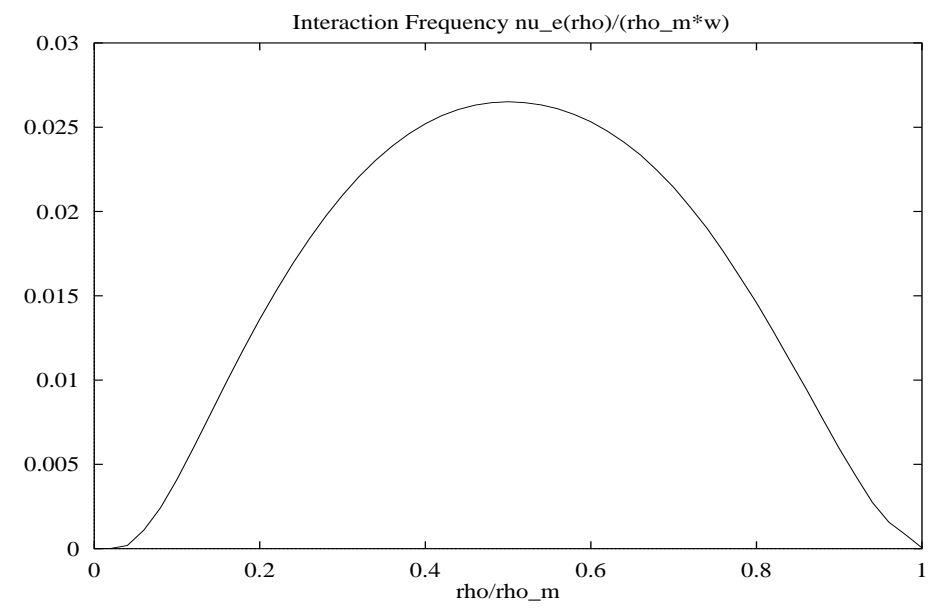




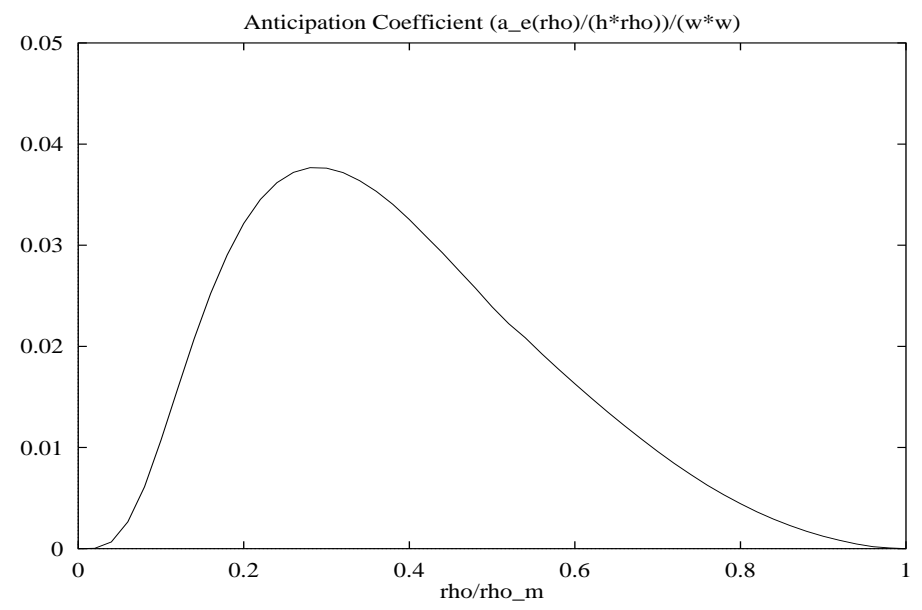


$\mathrm{F}(\mathrm{x}, \mathrm{v})$

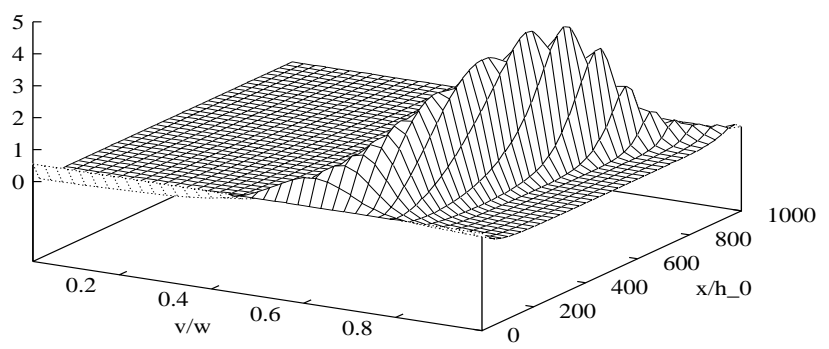


$\mathrm{F}(\mathrm{x}, \mathrm{v})$

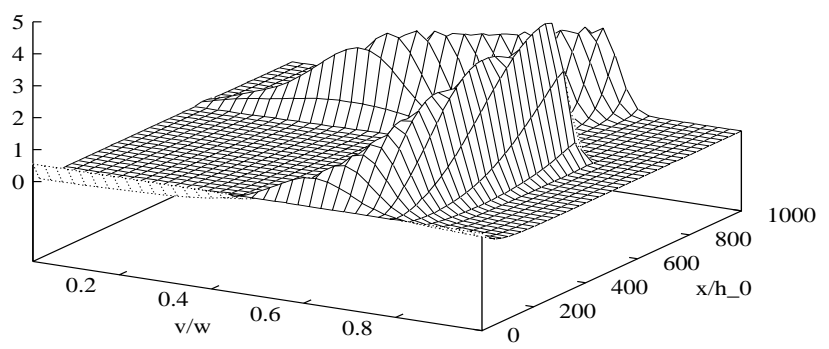


$\mathrm{F}(\mathrm{x}, \mathrm{v})$

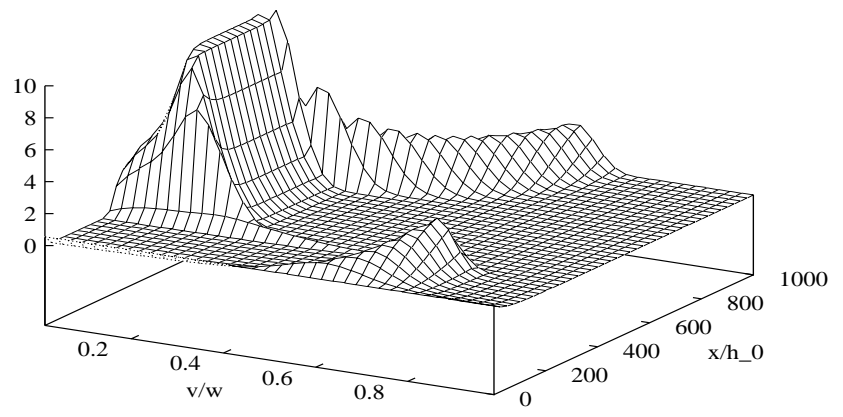




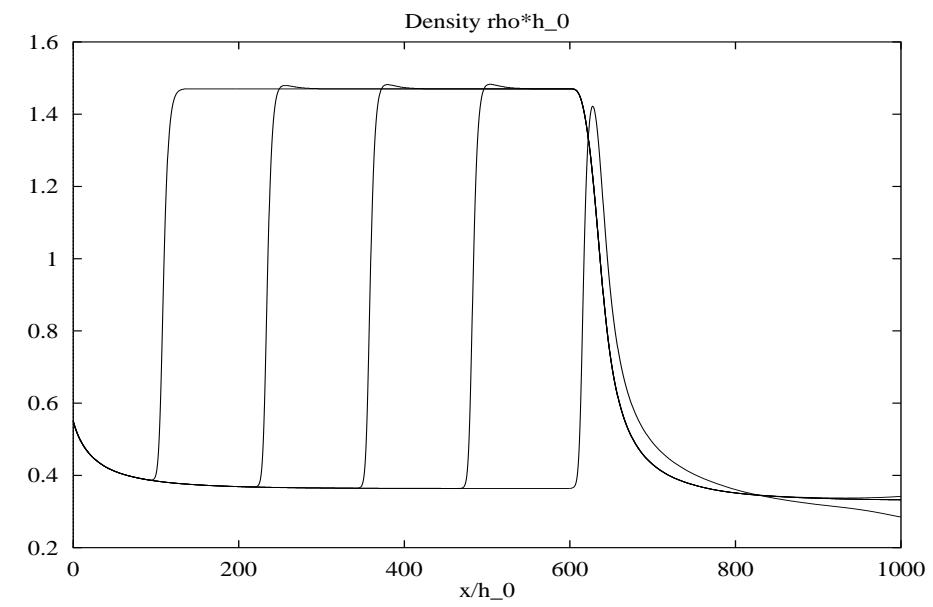




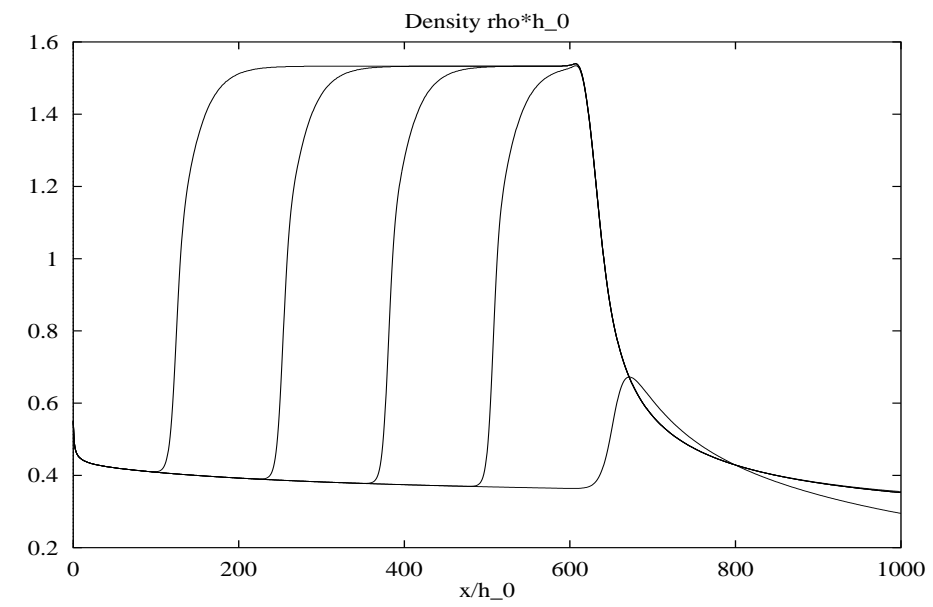




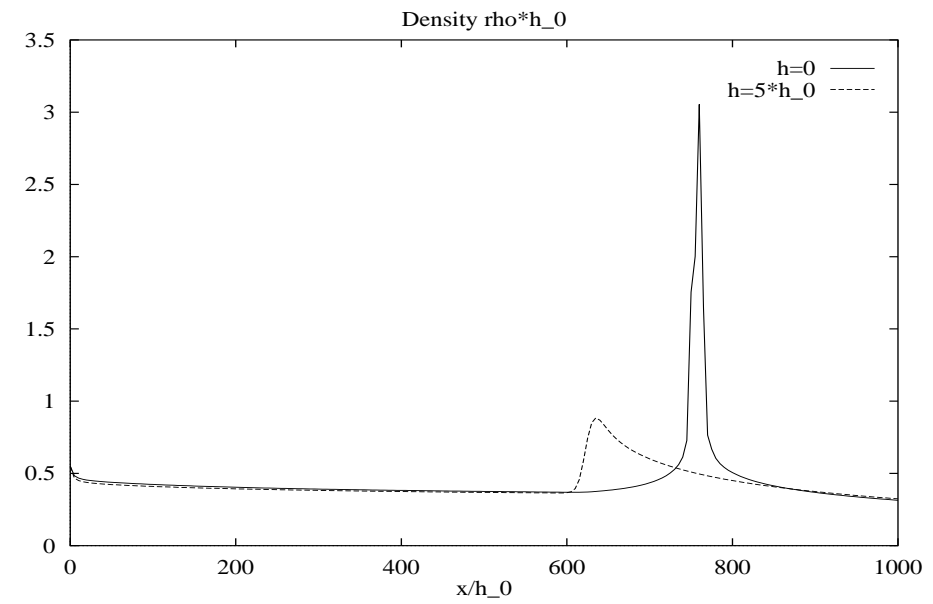

